\title{
VARIABILIDAD GENÉTICA EN SEMILLAS DE ÁRBOLES INDIVIDUALES DE Tectona grandis L.f. EN LA CONFORMACIÓN DE LOTES MEZCLADOS EN CÓRDOBA, COLOMBIA
}

\section{GENETIC VARIABILITY IN Tectona grandis L.f. SEEDS OF INDIVIDUAL TREES IN THE CONFORMATION OF MIXED SEEDLOTS IN CÓRDOBA, COLOMBIA}

\author{
Ender Correa ${ }^{1}$, Miguel Espitia $^{2}$, Hermes Araméndiz ${ }^{2}$, Olman Murillo ${ }^{3}$, Iván Pastrana ${ }^{4}$
}

${ }^{1}$ I.A. M.Sc., Facultad de Ciencias Agrícolas, Universidad de Córdoba, Montería, Colombia, endermanz@hotmail.com; ${ }^{2}$ I.A. Ph.D., Facultad de Ciencias Agrícolas, Universidad de Córdoba, Diagonal 50 \# 8-105 Barrio Villa del Rio Montería, Córdoba, mespitia37@hotmail.com; haramendiz@hotmail.com; ${ }^{3}$ I.F. Ph.D., Instituto Tecnológico de Costa Rica, olmuga@yahoo.es; ${ }^{4}$ I.A. M.Sc., Corpoica C.I. Motilonia Codazzi, Colombia, ijpv0710@hotmail.com

Rev. U.D.C.A Act. \& Div. Cient. 16(2): 379-389, Julio-Diciembre, 2013

\section{RESUMEN}

Para determinar el impacto del aporte individual de cada árbol en lotes mezclados, las implicaciones en la variabilidad genética de las futuras plantaciones y la estimación de parámetros genéticos para caracteres de germinación y de dimensiones de semillas, se estudiaron 14 lotes de semilla de árboles plus de Tectona grandis L.f., seleccionados en plantaciones comerciales del departamento de Córdoba. En una muestra de 100 semillas/lote, se estimaron las variables alométricas y los parámetros fisiológicos. El porcentaje de germinación (PG) presentó un ámbito de dispersión amplio, con valores desde $0 \%$ hasta $40 \%$; mientras que los caracteres alométricos, ancho y largo máximo (AS, LS) y la relación ancho/largo (RAL) mostraron poca variación. Los parámetros fisiológicos registraron una correlación genética significativa, con valores de $\mathrm{r}_{\mathrm{G}} \geq 0,91 * *$, mientras que los caracteres alométricos presentaron valores significativos, menores $\left(r_{\mathrm{G}} \leq 0,53 * *\right)$. Se registró una heredabilidad media familiar $\left(h^{2}\right) \geq 0,87$ en los caracteres investigados. Estos resultados reproducen la situación típica de programas semilleros, cuya información es importante para la obtención apropiada de lotes de semilla comercial, en mezcla de varios árboles; permite contribuir con la planeación y la conservación de la variabilidad genética y el manejo de las unidades productoras de germoplasma.

Palabras clave: Conservación, germoplasma, teca, parámetros genéticos, recursos fitogenéticos forestales.

\section{SUMMARY}

The aim of this research was to evaluate the impact of individual trees in mixed seed lots, its implications in genetic variability in future plantations, as well as, the estimation of their seed and germination genetic parameters in 14 individual plus tree seed lots of Tectona grandis L.f. from commercial plantations in Córdoba (Colombia). A sample of 100 seeds/lot was used to estimate allometric variables and physiological parameters. The germination percentage (GP) registered wide dispersion values from 0 to $40 \%$, while the allometric parameters maximum with and length (MW, ML), and with/length ratio (WLR), presented low variation. Among physiological parameters significative genetic correlations with values $\mathrm{r}_{\mathrm{G}} \geq 0.91 * *$ were registered. Meanwhile, genetic correlations among allometric parameters were also significative but with lower values, $r_{G} \leq 0.53 * *$. High mean family heritabilities $\left(\mathrm{h}^{2}\right) \geq 0.87$ were registered in all investigated traits. These results show the typical situations in seed programs therefore, this information is important for the proper constitution of commercial mixed seed lots. This knowledge contributes to the planning of genetic conservation and genetic variability in plantations, as well as to the management of germplasm production units.

Key words: Conservation, germoplasm, teak, genetic parameters, forest genetic resources. 


\section{INTRODUCCIÓN}

De cerca de 900 mil ha con aptitud forestal en Córdoba, solamente 30 mil están plantadas, con fines comerciales, con especies maderables nativas e introducidas, como Tectona grandis Linn. F, Gmelina arborea Roxb. y Acacia mangium Willd., que abarcan un 57,5\% del área total, lo que refleja la importancia de estas especies en la actividad forestal del departamento; para el 2030, se estima una superficie forestal plantada de 100 mil ha (CFC, 2011).

La variabilidad genética refleja su importancia al permitir amortiguar algunos efectos ambientales nocivos; además, da lugar a procesos genéticos, como el flujo de genes y la selección natural y reduce la deriva genética y endogamia (Namkoong, 1997; Boshier \& Amaral, 2004). Esta variabilidad, se reduce en plantaciones forestales establecidas a partir de clones o pocos genotipos, o si todo el programa de mejoramiento genético (PMG) se basa en un bajo número de árboles seleccionados (Schuhli \& Paludzyszyn-Filho, 2010; Lo et al. 2010). Por tanto, al planear la actividad forestal comercial, se debe garantizar una variabilidad genética mínima en campo (Murillo et al. 2012).

Rego et al. (2005) y Costa et al. (2009) indican que estudiar la variabilidad genética de lotes mixtos de semillas de árboles plus constituye una fase de pre-mejoramiento, que brinda información importante para establecer un PMG, que incluya la conservación de tal variabilidad en las plantaciones. Así mismo, Alba-Landa et al. (2007) sugieren la posibilidad de poder elegir, desde semillas y plántulas, cuáles serán las de mejor calidad para ambientes diferentes, como conocimiento básico, para selección temprana.

La calidad fisiológica de semillas se ha correlacionado con características, como coloración, tamaño, peso y contenido de humedad, entre otras (Rodríguez-Rivas et al. 2001; Aráoz et al. 2004; Niembro et al. 2007). En fitomejoramiento, las asociaciones entre caracteres de interés se evalúan por medio de correlaciones genotípicas, fenotípicas y ambientales; solamente la primera, se emplea para orientar PMG, por ser la única de naturaleza heredable; por ello, si dos caracteres presentan una correlación genética alta es posible seleccionar uno de ellos, a través de la selección del carácter asociado (Vallejo et al. 2010).

Entre los parámetros genéticos en poblaciones vegetales, se resaltan la heredabilidad y el coeficiente de variación genético. Ambos parámetros son buenos indicadores de variabilidad y de ganancia genética de los caracteres de interés en programas mejoramiento genético (Resende, 2007; Adewale et al. 2010; Shimelis \& Shiringani, 2010).

El presente estudio tuvo como objetivo principal determinar el efecto en la variabilidad genética de lotes individuales de semilla de árboles plus de T. grandis L.f. en la conformación de un lote mezclado. Además, se determinaron varios parámetros genéticos de la población y se obtuvieron correlaciones entre caracteres alométricos y fisiológicos de las semillas investigadas.

\section{MATERIALES Y MÉTODOS}

El estudio, se adelantó en el invernadero y laboratorio de Fitomejoramiento de la Universidad de Córdoba (Montería, Colombia), durante el 2011 y 2012. Se evaluaron 14 lotes familiares de semilla sexual de polinización libre de árboles plus de $T$. grandis L.f., seleccionados en diferentes plantaciones comerciales del departamento de Córdoba (Colombia), más una mezcla balanceada de estos lotes individuales. Dado que la teca es considerada una especie prevalentemente alógama (Dias et al. 2009; Schuhli \& Paludzyszyn-Filho, 2010), para los análisis estadísticos, se asumió que cada lote correspondió a una familia de medios hermanos.

En laboratorio, se determinaron variables alométricas de semilla: ancho máximo (AS), largo máximo (LS), relación ancho/largo (RAL) y peso fresco (PS). Se utilizó el diseño experimental completamente al azar, con 15 tratamientos y cuatro repeticiones de 25 semillas, a fin de analizar el efecto en la pérdida de variación genética al eliminar la semilla pequeña y de poco peso en lotes mezclados.

Los parámetros fisiológicos de semillas: porcentaje de germinación (PG), índice de velocidad de germinación (IVG), germinación diaria media (GDM), valor pico (Vp) y valor de germinación (VG), se evaluaron en invernadero utilizando un diseño experimental de bloques completos al azar con 15 tratamientos y cuatro repeticiones de 25 semillas. Debido a la dureza del mesocarpo de los frutos y la dificultad de extracción de sus semillas, que son pequeñas y delicadas, cada fruto no escarificado fue tratado como una semilla, criterio considerado común para varias especies forestales, de acuerdo a Figliolia et al. (1993) e ISTA (2009).

El tratamiento pre-germinativo consistió en cuatro ciclos de humedecimiento y secado a temperatura ambiente bajo sombra, con periodos intercalados de 24 horas por ciclo. Las semillas fueron sembradas en camas de germinación, con sustrato $100 \%$ de arena cuarsítica. La germinación fue valorada durante 55 días. El PG fue considerado como el porcentaje acumulado de semillas germinadas al final del ensayo; el IVG fue calculado mediante la fórmula recomendada por Maguire (1962):

$$
\mathrm{IVG}=\frac{P 1}{T 1}+\frac{P 2}{T 2}+\frac{P 3}{T 3}+\cdots \frac{P n}{T n}
$$


Donde: $\mathrm{P}_{1}, \mathrm{P}_{2}, \mathrm{P}_{3} \ldots, \mathrm{Pn}=$ número de plántulas normales, germinadas y completas en el primer, segundo, tercer y último conteo de la evaluación. $T_{1}, T_{2}, T_{3}, \ldots T_{n}=$ tiempo en días para cada germinación.

La GDM, se consideró como la relación entre el porcentaje acumulado de semillas germinadas al final del ensayo y el número de días desde la siembra al término del ensayo; el Vp como la GDM máxima alcanzada en el ensayo y el VG, correspondió al producto de la GDM con el VM (Czabator, 1962).

En todas las variables estudiadas, los resultados se ordenaron en categorías (alto, medio y bajo), a fin de poder relacionarlos con cada familia y su aporte al lote mezclado. Para el PG, las categorías fueron definidas de acuerdo a los siguientes rangos: Alto: $>66,6 \%$, Medio: entre 33,3 y 66,6\% y Bajo: $<33,3 \%$. En cuanto a las demás variables, se consideró al valor máximo obtenido en cada variable como el $100 \%$, a partir del cual, se establecieron, entonces, las tres categorías descritas anteriormente. Los datos fueron analizados mediante estadística paramétrica descriptiva, correlaciones fenotípicas, genéticas y ambientales y estimación de parámetros genéticos.

La estadística descriptiva, se consideró para analizar la variabilidad genética existente dentro y entre árboles en una población de teca, en este caso, de árboles plus. Los análisis de correlaciones, entre todos los caracteres evaluados, se usaron para identificar relaciones entre parámetros fisiológicos de germinación y características físicas de semillas, buscando sustentar mejores prácticas de manejo de lotes mezclados de semillas.

Los análisis de estadística descriptiva y las correlaciones, se realizaron mediante el uso del programa computacional GENES versión Windows (2004.2.1), desarrollado por Cruz (2004). Para estimar las correlaciones, el programa aplica las fórmulas clásicas de correlación:

a) Correlación fenotípica $\left(\mathrm{r}_{\mathrm{F}(\mathrm{XY})}\right) ; \mathrm{r}_{\mathrm{F}(\mathrm{XY})}=\mathrm{COV}_{\mathrm{F}(\mathrm{XY})} / \mathrm{S}_{\mathrm{F}(\mathrm{X})} \cdot \mathrm{S}_{\mathrm{F}(\mathrm{Y})}$

b) Correlación genética $\left(\mathrm{r}_{\mathrm{G}(\mathrm{XY})}\right) ; \mathrm{r}_{\mathrm{G}(\mathrm{XY})}=\mathrm{COV}_{\mathrm{G}(\mathrm{XY})} / \mathrm{S}_{\mathrm{G}(\mathrm{X})} \cdot \mathrm{S}_{\mathrm{G}(\mathrm{Y})}$

c) Correlación ambiental $\left(r_{E(X Y)}\right) ; r_{E(X Y)}=\operatorname{COV}_{E(X Y)} / S_{E(X)} \cdot S_{E(Y)}$

Dónde: $\mathrm{r}_{(\mathrm{XY})}$, $\mathrm{COV}_{(\mathrm{XY})}, \mathrm{S}(\mathrm{x})$ y $\mathrm{S}(\mathrm{y})$ son las correlaciones, covarianzas y las desviaciones estándar fenotípicas, genéticas y ambientales entre los caracteres X e Y, respectivamente.

Una vez estimados los coeficientes de correlación, se confirmó la significancia estadística para cada coeficiente de correlación, planteando la hipótesis nula: Ho: $\rho=0$ versus la hipótesis alterna: Ha: $\rho \neq 0$, mediante una prueba de $\mathrm{T}$, dada por la siguiente fórmula (Steel \& Torrie, 1980):

$$
T c=\frac{r \sqrt{(n-2)}}{\sqrt{\left(1-r^{2}\right)}}
$$

La T calculada (Tc): se comparó con una T tabular $(\mathrm{Tt})$, al nivel de significancia seleccionado: 0,05 y 0,01 y con (n 2) grados de libertad. La regla de decisión fue: sí Tc $\geq T t$, entonces el valor de $\rho$ es estadísticamente diferente de cero.

Los parámetros genéticos estimados fueron heredabilidad media familiar $\left(\mathrm{h}^{2}\right)$, exactitud de la selección de progenie (Acprog), coeficiente de variación genética aditiva individual (CVgi) y coeficiente de variación relativa (CVr). La estimación de estos parámetros, se realizó con el software Selegen-Re$\mathrm{ml} /$ Blup (Resende, 2006), utilizando el modelo 1 (diseño de bloques al azar, progenies de medios hermanos, varias plantas por parcela), para las variables alométricas y el modelo 19 (diseño de bloques al azar, evaluación de familias de medios hermanos y el conjunto de semillas/familia/repetición es la parcela), para los parámetros de calidad fisiológica:

$$
\begin{array}{ll}
\text { Modelo 1: } & \mathrm{y}=\mathrm{Xr}+\mathrm{Za}+\mathrm{Wp}+\mathrm{e} ; \\
\text { Modelo 19: } & \mathrm{y}=\mathrm{Xr}+\mathrm{Za}+\mathrm{e}
\end{array}
$$

Donde "y" es el vector de datos, "r" el vector de efectos de repetición sumados a la media general, "a" el vector de efectos genéticos aditivos individuales, "p" el vector de efectos de parcela y "e" el vector de errores residuales. Las mayúsculas representan las matrices de incidencia para los efectos referidos (Resende, 2006). Estos parámetros permite discutir el potencial de mejoramiento genético en caracteres alómetricos y fisiológicos de la semilla, para un mejor manejo de lotes mezclados de semillas.

Finalmente, con los valores de germinación de cada uno de los 14 lotes de semilla, se construyó una población hipotética de 1.111 plántulas equivalente a 1 ha plantada a $3 \mathrm{~m} \times 3 \mathrm{~m}$, donde cada familia aportaría el mismo número de plántulas, a fin de analizar y discutir la variabilidad genética real de esta plantación.

\section{RESULTADOS Y DISCUSIÓN}

El grupo de variables fisiológicas de las semillas evidenció diferencias estadísticas significativas $(\operatorname{Pr}<0,01)$ entre lotes, indicando que al menos uno de los lotes presentó promedio estadísticamente diferente para PG, IVG, VG, GDM y Vp (Tabla 1). Resultados similares han sido reportados por Vieira et al. (2009), Costa et al. (2009), Dias et al. (2009), Rocha et al. (2011), Ferreira et al. (2012), Silva et al. (2012) y Gallo et al. (2012). 
Tabla 1. Parámetros fisiológicos, alométricos y peso de semillas de 14 árboles plus de Tectona grandis L.f., seleccionados en el departamento Córdoba, Colombia.

\begin{tabular}{|c|c|c|c|c|c|c|c|c|c|}
\hline Árbol & $\begin{array}{c}\text { PG } \\
(\%)\end{array}$ & IVG & VG & GDM & VP & $\begin{array}{c}\text { AS } \\
(\mathrm{cm})\end{array}$ & $\begin{array}{c}\text { LS } \\
(\mathrm{cm})\end{array}$ & RAL & $\begin{array}{c}\text { PS } \\
(\mathrm{g})\end{array}$ \\
\hline \multicolumn{7}{|c|}{ Fisiológicos } \\
\hline 1 & 7,0 & 0,36 & 0,05 & 0,13 & 0,29 & 1,40 & 1,32 & 1,06 & 0,90 \\
\hline 2 & 0,0 & 0,00 & 0,00 & 0,00 & 0,00 & 1,21 & 1,14 & 1,07 & 0,55 \\
\hline 3 & 5,0 & 0,29 & 0,03 & 0,09 & 0,27 & 1,35 & 1,38 & 0,98 & 0,62 \\
\hline 4 & 16,0 & 0,64 & 0,13 & 0,29 & 0,44 & 1,60 & 1,35 & 1,19 & 0,93 \\
\hline 5 & 29,0 & 1,85 & 0,75 & 0,53 & 1,35 & 1,48 & 1,24 & 1,20 & 0,65 \\
\hline 6 & 17,0 & 0,60 & 0,16 & 0,31 & 0,37 & 1,48 & 1,36 & 1,10 & 0,75 \\
\hline 7 & 20,0 & 1,53 & 0,40 & 0,36 & 1,12 & 1,24 & 1,30 & 0,96 & 0,57 \\
\hline 8 & 13,0 & 1,21 & 0,27 & 0,24 & 0,86 & 1,34 & 1,30 & 1,04 & 0,64 \\
\hline 9 & 22,0 & 0,70 & 0,25 & 0,40 & 0,47 & 1,39 & 1,26 & 1,10 & 0,81 \\
\hline 10 & 4,0 & 0,11 & 0,01 & 0,07 & 0,09 & 1,37 & 1,39 & 0,99 & 0,76 \\
\hline 11 & 9,0 & 0,58 & 0,09 & 0,16 & 0,49 & 1,30 & 1,27 & 1,03 & 0,66 \\
\hline 12 & 40,0 & 3,76 & 1,97 & 0,73 & 2,61 & 1,32 & 1,28 & 1,04 & 0,62 \\
\hline 13 & 18,0 & 1,65 & 0,41 & 0,33 & 1,19 & 1,23 & 1,21 & 1,02 & 0,48 \\
\hline 14 & 6,0 & 0,51 & 0,07 & 0,11 & 0,42 & 1,27 & 1,28 & 1,00 & 0,62 \\
\hline MBS & 19,0 & 1,33 & 0,36 & 0,35 & 0,86 & 1,33 & 1,32 & 1,01 & 0,62 \\
\hline SIGEST. & $* *$ & $* *$ & $* *$ & $* *$ & $* *$ & $\mathrm{~ns}$ & $\mathrm{~ns}$ & $\mathrm{~ns}$ & $\mathrm{~ns}$ \\
\hline MG & 15,0 & 1,01 & 0,33 & 0,27 & 0,72 & 1,35 & 1,29 & 1,05 & 0,68 \\
\hline Min & 0,00 & 0,00 & 0,00 & 0,00 & 0,00 & 1,21 & 1,14 & 0,96 & 0,48 \\
\hline Max & 40,0 & 3,76 & 1,97 & 0,73 & 2,61 & 1,60 & 1,39 & 1,20 & 0,93 \\
\hline CV (\%) & 70,3 & 94,4 & 150,9 & 70,3 & 91,2 & 7,8 & 5,1 & 6,6 & 18,7 \\
\hline CB (\%) & 93,3 & 66,7 & 86,7 & 40,0 & 73,3 & 0 & 0 & 0 & 0 \\
\hline CM (\%) & 6,7 & 26,7 & 6,7 & 46,7 & 20,0 & 0 & 0 & 0 & 26,7 \\
\hline CA (\%) & 0,0 & 6,7 & 6,7 & 13,3 & 6,7 & 100 & 100 & 100 & 73,3 \\
\hline
\end{tabular}

PG: porcentaje de germinación; IVG: índice de velocidad de germinación; VG: valor de germinación; GDM: germinación diaria media; Vp: valor pico; AS: ancho de semilla; LS: largo de semilla; RAL: relación ancho/largo de semilla; PS: peso de semilla; MBS: mezcla balanceada de semillas; SIGEST: significancia estadística; MG: media general; Min: valor mínimo; Max: valor máximo; CV: coeficiente de variación; CB: categoría baja; CM; categoría media; CA: categoría alta.

Los porcentajes de germinación (PG) variaron desde $0 \%$ hasta $40 \%$ ( $\bar{x}=15 \%$; $C V=70,3 \%$ ), obteniéndose que el $93,3 \%$ y el $6,7 \%$ de los árboles presentan PG bajos y medios, respectivamente (Tabla 1). El amplio ámbito de dispersión de los PG podría ser el resultado de diferencias genéticas entre las familias, estado de madurez de la semilla, manejo y almacenamiento de la semilla de los diferentes árboles, además de los efectos ambientales, como factores bióticos y abióticos, bajo los cuales, se produjeron las semillas (Edwards,
1980; Pastorino \& Gallo, 2000). Es importante conocer el origen de las semillas, ya que se puede presentar variación entre y dentro de una misma población, familia o procedencia (Hoppe et al. 2004). Además, algunas características intrínsecas de las semillas, como sustancias hormonales y sustancias inhibidoras no hormonales, también pueden influir en su respuesta germinativa (Aguiar et al. 1993; Venable \& Pake, 1999). 
Los PG obtenidos en el estudio son inferiores a los reportados por Costa et al. (2009), al estudiar 60 lotes familiares de semillas de teca en Brasil (Municipio de Alegre), quienes reportan PG entre el 5 y $94 \%$. Las diferencias en los resultados, se puede deber a la cantidad y a las diferencias genéticas de los lotes (14 vs 60) e, igualmente, al balance entre factores intrínsecos y extrínsecos, que afectan, de manera primordial, los procesos de reparación y los mecanismos deletéreos del metabolismo en la semillas.

El período de viabilidad de las semillas es extremadamente variable y está determinado genéticamente, aunque factores ambientales y condiciones de almacenamiento tienen un efecto decisivo en la longevidad de la semilla (Carvalho \& Nakagawa, 1998). Cabe anotar que las semillas de T. grandis se caracterizan por presentar una germinación lenta e irregular (Rocha et al. 2011) y los porcentajes de germinación varían considerablemente, con valores entre 10 y $80 \%$ (Chaves \& Fonseca, 1991), 4 al 78\% (Vieira et al. 2009) y 28 al $73 \%$ (Dias et al. 2009). Por otra parte, el 46,7\% de los árboles plus de teca, evaluados en este estudio, provienen del municipio de Puerto Libertador (Córdoba), que es clasificado como bosque húmedo tropical (bh-T) (Palencia et al. 2006). En este sentido, la alta humedad relativa ( $\geq 80 \%$ ) y la temperatura $\left(\geq 27^{\circ} \mathrm{C}\right)$ favorecen el envejecimiento acelerado de las semillas, dado que estas variables afectan los procesos bioquímicos relacionados con la germinación (Araméndiz et al. 2007). Además, estas semillas pueden bajar su PG hasta un $15 \%$ después de un año de almacenamiento (Fonseca, 2004).

Las características alométricas de las semillas AS, LS, RAL y PS no presentaron diferencias significativas entre lotes de semilla. Esto señala poca variabilidad y se evidencia, a través de valores de CV menores del 19\%. Los valores promedios en este grupo de variables permiten clasificar a todos los árboles en la categoría alta (CA); excepto para PS, donde solamente el $73,3 \%$ de los lotes de semilla estuvo en la categoría alta y el 26,7\% restante, en la categoría media (CM). El AS osciló entre 1,21 y $1,60 \mathrm{~cm}$; LS entre 1,14 y $1,39 \mathrm{~cm}$, RAL entre 0,96 y 1,20 y el PS entre 0,48 y 0,93g (Tabla 1 ). Estas dimensiones son próximas a las reportadas por Suri (1984) y Caldeira et al. (2000), con tamaño de semillas entre 1,12 y $1,57 \mathrm{~cm} ; 1,25$ a $1,60 \mathrm{~cm}$ y $1,0 \mathrm{~cm}$, respectivamente. Estos resultados permiten determinar que, para los lotes evaluados, el tamaño de la semilla no traería mayores problemas en lotes mezclados, ya que hubo poca variación entre árboles y, por tanto, no serían eliminadas familias enteras al desecharse las semillas de menor tamaño.

Correlaciones fenotípicas $\left(r_{\mathrm{F}}\right)$, genéticas $\left(r_{\mathrm{G}}\right)$ y ambientales $\left(r_{\mathrm{E}}\right)$ : La tabla 2 presenta los valores de las correlaciones fenotípicas, genéticas y ambientales. Se observa que para los parámetros de calidad fisiológica (PG, IVG, VG, GDM y Vp), las $r_{G}$ fueron altamente significativas, positivas $\left(r_{G} \geq 0,91 * *\right)$ y ligeramente mayores a las $r_{F}\left(r_{F} \geq 0,87 * *\right)$. Lo anterior sugiere una $r_{E}$ importante, señalando que los caracteres asociados son beneficiados o perjudicados por las mismas variaciones ambientales y que, además, los genes que controlan tales caracteres presentan efectos pleiotrópicos o existe falta de equilibrio en ligamiento.

Otros autores reportan resultados similares en teca y sugieren que mayores IVG se asocian a los lotes de semilla con mayor PG (Indira et al. 2000; Vieira et al. 2009; Dias et al. 2009). En Hevea brasiliensis, se reportan correlaciones lineales simples (fenotípicas) de $r=0,95^{* *}$, entre PG con el IVG (Moreno et al. 2006). Las correlaciones fenotípicas y genéticas fueron iguales para el PG vs. GDM y AS vs. RAL. En estas variables, se puede considerar un efecto ambiental nulo sobre la asociación real entre los caracteres, ya que la GDM y RAL son variables compuestas, calculadas a partir de datos o mediciones del PG y del AS, respectivamente.

No se obtuvieron $\mathrm{r}_{\mathrm{G}}$ significativas entre los parámetros fisiológicos y los rasgos alométricos AS y LS. Ello, posiblemente, indique que son caracteres con control genético diferente, dado que, además la $\mathrm{r}_{\mathrm{E}}$, tampoco fue significativa. El PS presentó asociación baja, inversa y significativa con el IVG $\left(\mathrm{r}_{\mathrm{G}}=\right.$ $-0,38 * *), V G\left(r_{G}=-0,28 * *\right)$ y VP $\left(r_{G}=-0,40 * *\right)$, sugiriendo que semillas pequeñas tienden a presentar mejores parámetros fisiológicos. Este resultado se podría considerar riesgoso, en caso de constituir un lote mezclado y luego eliminar la semilla pequeña, ello podría incurrir en deriva o erosión genética y, por tanto, se reduciría su variabilidad genética.

El tamaño y peso de semillas de un mismo árbol, por lo general, está asociado con una mayor germinación y desempeño de las plántulas, en razón a que semillas grandes y pesadas contienen mayores cantidades de carbohidratos en el endospermo o cotiledones, que refleja la disponibilidad de una mayor fuente de energía, para estimular la germinación, emergencia, supervivencia y crecimiento de las plántulas (Khurana \& Singh, 2001). Estas correlaciones han sido observadas en varios trabajos (Leishman et al. 2000; Tenorio-Galindo et al. 2008; Huerta-Paniagua \& Rodríguez-Trejo, 2011); sin embargo, otras investigaciones no reportan tales asociaciones (Alba-Landa et al. 2007), pero la variación en el tamaño promedio de semillas entre árboles latifoliados está explicado por el fenómeno de la doble fertilización, donde existe una mayor participación materna en el endospermo $\left(2 \mathrm{n} \uparrow+1 \mathrm{n}{ }^{\hat{}}\right)$, por lo que su tasa de germinación promedio no guarda relación con el tamaño promedio de su semilla (Murillo, 1998).

Parámetros genéticos: Los parámetros de calidad fisiológica registraron valores de heredabilidad media familiar $\left(\mathrm{h}^{2}\right)$ altos, entre 0,87 y 0,92 (Tabla 3), siendo coincidentes con 
Tabla 2. Correlaciones fenotípicas $\left(\mathrm{r}_{\mathrm{F}}\right)$, genéticas $\left(\mathrm{r}_{\mathrm{G}}\right)$ y ambientales $\left(\mathrm{r}_{\mathrm{E}}\right)$ para parámetros de calidad fisiológica y dimensiones de semillas de árboles plus de T. grandis, seleccionados en el departamento de Córdoba, Colombia.

\begin{tabular}{|c|c|c|c|c|c|c|c|c|c|}
\hline & $r^{\prime} s$ & IVG & VG & GDM & $V p$ & AS & LS & RAL & PS \\
\hline \multirow{3}{*}{ PG } & $\mathrm{r}_{\mathrm{F}}$ & $0,90 * *$ & $0,87 * *$ & $1,00 * *$ & $0,89 * *$ & 0,21 & $-0,08$ & $0,32 *$ & $-0,09$ \\
\hline & $r_{G}$ & $0,93 * *$ & $0,91 * *$ & $1,00 * *$ & $0,93 * *$ & 0,23 & $-0,09$ & $0,34 * *$ & $-0,10$ \\
\hline & $r_{E}$ & $0,72 * *$ & $0,63 * *$ & $1,00 * *$ & $0,56 * *$ & 0,04 & 0,02 & 0,02 & 0,09 \\
\hline \multirow{3}{*}{ IVG } & $r_{F}$ & & $0,96 * *$ & $0,90 * *$ & $0,99 * *$ & $-0,09$ & $-0,16$ & 0,04 & $-0,35 * *$ \\
\hline & $r_{G}$ & & $0,98 * *$ & $0,93 * *$ & $1,00 * *$ & $-0,09$ & $-0,17$ & 0,05 & $-0,38 * *$ \\
\hline & $r_{E}$ & & $0,83 * *$ & $0,72 * *$ & $0,94 * *$ & 0,04 & 0,10 & $-0,04$ & 0,20 \\
\hline \multirow{3}{*}{ VG } & $r_{F}$ & & & $0,87 * *$ & $0,95 * *$ & $-0,04$ & $-0,15$ & 0,09 & $-0,25$ \\
\hline & $r_{G}$ & & & $0,91 * *$ & $0,97 * *$ & $-0,04$ & $-0,18$ & 0,11 & $-0,28^{*}$ \\
\hline & $\mathrm{r}_{\mathrm{E}}$ & & & $0,63 * *$ & $0,82 * *$ & 0,00 & 0,25 & $-0,20$ & 0,20 \\
\hline \multirow{3}{*}{ GDM } & $\mathrm{r}_{\mathrm{F}}$ & & & & $0,89 * *$ & 0,21 & $-0,08$ & $0,32 *$ & $-0,09$ \\
\hline & $r_{G}$ & & & & $0,93 * *$ & 0,23 & $-0,09$ & $0,34 * *$ & $-0,10$ \\
\hline & $r_{E}$ & & & & $0,56 * *$ & 0,04 & 0,02 & 0,02 & 0,09 \\
\hline \multirow{3}{*}{ Vp } & $\mathrm{r}_{\mathrm{F}}$ & & & & & $-0,10$ & $-0,17$ & 0,03 & $-0,37 * *$ \\
\hline & $r_{G}$ & & & & & $-0,11$ & $-0,19$ & 0,04 & $-0,40 * *$ \\
\hline & $r_{E}$ & & & & & 0,04 & 0,20 & $-0,12$ & 0,24 \\
\hline \multirow{3}{*}{ AS } & $\mathrm{r}_{\mathrm{F}}$ & & & & & & $0,50 * *$ & $0,76 * *$ & $0,78 * *$ \\
\hline & $r_{G}$ & & & & & & $0,51 * *$ & $0,76 * *$ & $0,79 * *$ \\
\hline & $\mathrm{r}_{\mathrm{E}}$ & & & & & & $0,49 * *$ & $0,70 * *$ & $0,37 * *$ \\
\hline \multirow{3}{*}{ LS } & $\mathrm{r}_{\mathrm{F}}$ & & & & & & & $-0,17$ & $0,50 * *$ \\
\hline & $r_{G}$ & & & & & & & $-0,17$ & $0,51 * *$ \\
\hline & $r_{E}$ & & & & & & & $-0,29 *$ & 0,24 \\
\hline \multirow{3}{*}{ RALs } & $\mathrm{r}_{\mathrm{F}}$ & & & & & & & & $0,50 * *$ \\
\hline & $r_{G}$ & & & & & & & & $0,51 * *$ \\
\hline & $r_{E}$ & & & & & & & & 0,23 \\
\hline
\end{tabular}

PG: porcentaje de germinación (\%); IVG: índice de velocidad de germinación; VG: valor de germinación; GDM: germinación diaria media; Vp: valor pico; AS: ancho de semilla (cm); LS: largo de semilla (cm); RAL: relación ancho/largo de semilla; PS: peso fresco de semilla (g); *significativo (5\%); ** altamente significativo (1\%).

las $\mathrm{r}_{\mathrm{G}} \mathrm{y}_{\mathrm{F}}$ registradas en la tabla 2. Estos resultados concuerdan con lo reportado por Vallejo et al. (2010), debido a que caracteres con alta heredabilidad, presentan, por lo general, valores de correlación fenotípica y genética estrechas o similares.

La heredabilidad media familiar de PG obtenida fue de 0,87 , cuyo valor es similar a la reportada para este mismo carácter $\left(\mathrm{h}^{2}=0,89\right)$ por Costa et al. (2009) en teca y Rego et al. (2005), en semillas de Albizia lebbeck $\left(h^{2}=0,79\right)$. Altos valores de heredabilidad indican bajos efectos ambientales en la expresión de los caracteres, control genético por genes mayores y eficiencia en procesos de selección. De igual forma, para los caracteres considerados, la exactitud (Acprog) en la estimación de parámetros genéticos (Tabla 3) fue alta $(\geq 0,93)$. Al respecto, Resende et al. (1995), Costa et al. (2000) y Costa et al. (2005) señalan que a mayor magnitud en Acprog, mayor será la confiabilidad de los parámetros genéticos obtenidos, así como para su interpretación y selección de materiales. 
Tabla 3. Parámetros genéticos estimados para caracteres alométricos y de calidad fisiológica en semillas de T. grandis, en el departamento de Córdoba.

\begin{tabular}{|l|c|c|c|c|c|c|c|c|c|}
\hline & PG & IVG & VG & GDM & Vp & AS & LS & RAL & PS \\
\hline $\mathrm{h}^{2}$ & 0,87 & 0,92 & 0,88 & 0,87 & 0,91 & 0,97 & 0,96 & 0,97 & 0,98 \\
\hline Acprog & 0,93 & 0,96 & 0,94 & 0,93 & 0,95 & 0,98 & 0,98 & 0,98 & 0,99 \\
\hline CVgi \% & 131,09 & 180,89 & 284,02 & 130,8 & 174,02 & 15,42 & 10,07 & 12,94 & 36,96 \\
\hline CVr & 1,29 & 1,67 & 1,37 & 1,29 & 1,59 & 2,77 & 2,45 & 2,71 & 3,55 \\
\hline
\end{tabular}

PG: porcentaje de germinación; IVG: índice de velocidad de germinación; VG: valor de germinación; GDM: germinación diaria media; Vp: valor pico; AS: ancho de semilla; LS: largo de semilla; RAL: relación ancho/largo de semilla; PS: peso fresco de semilla; $h^{2}$ : heredabilidad media familiar; Acprog: exactitud de la selección de progenie; CVgi: coeficiente de variación genética aditiva individual; CVr: coeficiente de variación relativa.

Para los parámetros de calidad fisiológica, el coeficiente de variación genética aditiva individual (CVgi) fue alto en la mayoría de los rasgos evaluados, siendo mayores a 130,8\%. De acuerdo con Costa et al. (2009), estos CVgi expresan la cantidad de variación genética individual existente. Por tanto, valores altos del CVgi denotan que, en futuras evaluaciones en campo, podría haber mayor expresión de variación genética para otros caracteres asociados, con buenas perspectivas de variabilidad, para ser aprovechada a lo largo de un programa de mejoramiento genético.

En cuanto al parámetro coeficiente de variación relativa (CVr), el cual, es la relación entre el CV genético y el CV fenotípico, los valores obtenidos en este parámetro oscilaron entre 1,29 y 1,67 (Tabla 3). Resultados similares reportan Costa et al. (2009), para este grupo de parámetros fisiológicos, en teca. Según Resende \& Duarte (2007), estos CVr indican una buena oportunidad de ganancia genética al seleccionar en lotes de semillas, por tanto, el carácter tiene potencial para ser mejorado genéticamente.

Lotes mezclados de semillas: En una mezcla balanceada (MBS) ordinaria o convencional, se asume que cada lote de semillas (familias) aporta igual cantidad de semilla al lote compuesto o mezclado; sin embargo, bajo este parámetro y sin considerar la calidad fisiológica de cada semilla, lotes con alta germinación (por su valor adaptativo) estarían realmente aportando varias veces más plántulas efectivas que aquellos que presentan baja germinación, tal y como reporta Murillo (1998), en un estudio con lote mezclado, a partir de 50 familias de Alnus acuminata.

Este estudio determinó (Tabla 4) que lotes de semillas pertenecientes a categorías de germinación media aportan en promedio 2,8 veces (200/71) más plántulas que lotes de semilla de baja germinación dentro del lote originado de la mezcla de igual número de semilla (MBS ordinaria). Mientras que, en la mezcla balanceada ajustada (MBS ajustada) al PG, el número de plántulas a obtener por lote de semillas sería teóricamente el mismo para todas las familias.

Este tipo de lote de semilla mezclado con base a un mismo número de semillas por árbol, evidencia una participación desigual de las familias de semilla en un hipotético lote balanceado de manera convencional, dado que no todas las semillas de cada árbol presentan la misma capacidad germinativa. Por ello, es muy probable que después de un costoso y laborioso esfuerzo en el trabajo de campo, donde se ha colectado semilla de un número representativo de árboles, la composición o la variabilidad de la población de plántulas que se obtendría al final de la fase de vivero para llevar a plantación, podría representar un número muy inferior de individuos a los que se les colectó la semilla (Davidson et al. 1996; Murillo, 1998; Rocha et al. 2011; Ferreira et al. 2012 y Silva et al. 2012).

Fofana et al. (2009) señalan que en teca la drástica reducción de la variabilidad genética se debe, entre otros aspectos, a la propagación vegetativa, basada en los árboles existentes en las plantaciones o con semillas mezcladas de pocos árboles provenientes de estas fuentes. Así también, es común que se presenten en las plantaciones individuos con mayor producción de semilla que otros. Por ello, autores como Varghese et al. (2006) proponen poner límites a la cantidad de semillas que pueden ser recogidos por árbol (genotipo), lo cual, podría ser útil en el control de la sobre-representación de los clones altamente reproductivos, aumentando, con esto, la variabilidad genética del lote mezclado.

Con base en los resultados obtenidos en este estudio, se puede inferir lo siguiente: i) la eliminación o descarte de semillas pequeñas o de poco peso en un lote mezclado, podría generar un efecto negativo sobre la variabilidad genética y la 
Tabla 4. Distribución de lotes de semilla de acuerdo con su porcentaje de germinación y su contribución hipotética (si se mezclara la semilla), a una producción conjunta aproximada de 1.111 plántulas requeridas, para el establecimiento de una hectárea de T. grandis, sembrada a $3 \mathrm{~m}$ x $3 \mathrm{~m}$.

\begin{tabular}{|c|c|c|c|c|c|c|c|c|c|c|c|}
\hline \multirow{3}{*}{$\begin{array}{l}\text { Catego- } \\
\text { rías de } \\
\text { germi- } \\
\text { nación }\end{array}$} & & & & \multicolumn{4}{|c|}{ MBS ordinaria } & \multicolumn{4}{|c|}{ MBS ajustada } \\
\hline & \multicolumn{3}{|c|}{$\begin{array}{l}\text { Lotes por categoría de } \\
\text { germinación }\end{array}$} & \multicolumn{2}{|c|}{$\begin{array}{l}\text { +Contribución de } \\
\text { plántulas/catego- } \\
\text { ría de germinación }\end{array}$} & \multicolumn{2}{|c|}{$\begin{array}{l}\text { +Contribución } \\
\text { media de plántu- } \\
\text { las/lote de semi- } \\
\text { llas }\end{array}$} & \multicolumn{2}{|c|}{$\begin{array}{l}{ }^{++} \text {Contribución de } \\
\text { plántulas/catego- } \\
\text { ría de germinación }\end{array}$} & \multicolumn{2}{|c|}{$\begin{array}{c}{ }^{++} \text {Contribución de } \\
\text { plántulas/lote de } \\
\text { semillas }\end{array}$} \\
\hline & No. & $\%$ & $\begin{array}{l}\text { Acumu- } \\
\text { lado }\end{array}$ & No. & $\%$ & No. & $\%$ & No. & $\%$ & No. & $\%$ \\
\hline $\begin{array}{l}\text { Baja: } \\
<33,3 \%\end{array}$ & 14 & 93,3 & $\begin{array}{c}14 \\
(93,3 \%)\end{array}$ & 925 & 82,2 & 71 & 6,3 & 1040 & 92,9 & 80 & 7,1 \\
\hline $\begin{array}{l}\text { Media: } \\
33,33 \% \text { - } \\
66,6 \%\end{array}$ & 1 & 6,7 & $\begin{array}{c}15 \\
(100 \%)\end{array}$ & 200 & 17,8 & 200 & 17,8 & 80 & 7,1 & 80 & 7,1 \\
\hline $\begin{array}{l}\text { Alta: } \\
>66,6 \%\end{array}$ & 0 & & & & & & & & & & \\
\hline Total & 15 & 100 & & 1125 & 100 & & & 1120 & 100 & & \\
\hline
\end{tabular}

MBS ordinaria: mezcla balanceada de semillas basada en la contribución de igual número de semillas por lote; MBS ajustada: mezcla balanceada de semillas basada en la contribución de un número de semillas por lote, de acuerdo a su porcentaje de germinación; + para una producción conjunta hipotética de 1125 plántulas en base a 500 semillas por lote; ++ para una producción conjunta hipotética de 1120 plántulas en base a la obtención de 80 plántulas por lote.

representatividad de los individuos de interés a nivel de vivero y futura plantación; ii) la creación de lotes semillas mezclados con base en la capacidad germinativa de la semilla de cada uno de los individuos que lo componen (árboles o clones), contribuye a la conservación de los recursos genéticos forestales y variabilidad genética de las plantaciones comerciales y iii) los caracteres fisiológicos y alométricos de semilla de teca investigados, muestran una heredabilidad o control genético alto, que demuestran un potencial importante para futuros programas de mejoramiento genético.

Conflicto de intereses: El manuscrito fue preparado y revisado con la participación de todos los autores, quienes declaramos que no existe ningún conflicto de intereses, que ponga en riesgo la validez de los resultados presentados.

\section{BIBLIOGRAFÍA}

1. ADEWALE, B.D.; OKONJI, C.; OYEKANMI, A.A.; AKINTOBI, D.A.; AREMU, C.O. 2010. Genotypic variability and stability of some grain yield components of cowpea. African J. Agr. Res. 5(9):874-880.

2. AGUIAR, I.; PIÑA-RODRIGUES, F.; FIGLIOLIA, M. 1993. Sementes Florestais Tropicais. Abrates, Brasília, Distrito Federal. 350p.

3. ALBA-LANDA, J.; RAMÍREZ-GARCÍA, E.; APARICIORENTERÍA, A. 2007. Correlación de semillas y plántulas de Pinus teocote Schl. Et Cham. de tres procedencias del estado de Veracruz, México. Foresta Veracruzana. 9(1):23-27. 
4. ARAMÉNDIZ, H.; CARDONA, C.; JARMA, A.; ROBLES, J.; MONTALVÁN, R. 2007. Efectos del almacenamiento en la calidad fisiológica de la semilla de berenjena (Solanum melongena L.). Agr. Col. 25(1):104-112.

5. ARÁOZ, S.; LONGO, O.; KARLIN, O. 2004. Germinación de semillas de Zizyphus mistol Grisebach III. Correlaciones paramétricas del tamaño y peso de drupas, endocarpos y semillas con la germinación y el vigor. Multequina. 13:51-56.

6. BOSHIER, D.; AMARAL, W. 2004. Amenazas a los ecosistemas silvícolas y desafíos para la conservación y uso sostenible de los recursos genéticos silvícolas. En: Vinceti, B.; Amaral, W.; Meilleur, B. (eds.) Desafíos de la ordenación de los recursos genéticos silvícolas para contribuir a la subsistencia: ejemplos de Argentina y Brasil. Ed. IPGRI, (Roma). p.7-32.

7. CADENA FORESTAL DE CÓRDOBA -CFC-. 2011. Acuerdo regional de competitividad: Cadena forestal madera, muebles y productos de madera del departamento de Córdoba 2011-2030.55p.

8. CALDEIRA, S.; CALDEIRA, S.; DE MENDOÇA, E.; DINIZ, N. 2000. Caracterização e avaliação da qualidade dos frutos de teca (Tectona grandis L.f.) produzidos no Mato Grosso. Rev. Bras. Sementes. 22(1):216224.

9. CARVALHO, N.M.; NAKAGAWA, J. 1998. Sementes: ciência, tecnologia e produção. $3^{\mathrm{a}}$ ed. Fundação Cargill. Campinas (Brasil). 424p.

10. CHAVES, E.; FONSECA, W. 1991. Teca, Tectona grandis L.f., especie de árbol de uso múltiple en América Central. Informe Técnico 179. Turrialba, Costa Rica: Centro Agron. Trop. Invest. y Enseñanza. 47p.

11. COSTA, R.; RESENDE, M.; ARAÚJO, A.; GONÇALVES, P.; SILVA, M. 2000. Maximization of genetic gain in rubber tree (Hevea) breeding with effective size restriction. Gen. Mol. Biol. 23(2):457-462.

12. COSTA, R.; GONÇALVES, P.; OLIVEIRA, L.; ARRUDA, E.; ROA, R.; MARTINS, W. 2005. Variabilidade genética e estimativas de heredabilidade para o carácter germinação em matrizes de Hevea brasiliensis. Rev. Floresta e Ambiente. 12(1):74-76.

13. COSTA, R.; CHICHORRO, J.; RESENDE, M.; ROA, R.; COTTA, T.; CEZANA, D. 2009. Variabilidade genética para o carácter germinação em matrizes de teca, no Município de Alegre, ES. Pesq. Floresta Bras. 59:57-61.
14. CRUZ, C. 2004. Programa genes. Versão Windows. Aplicativo computacional em genética e estatística. Universidade Federal de Viçosa. Disponible desde Internet en: http:///www.ufv.br/dbg/genes/genes.htm (con acceso 05/07/2007).

15. CZABATOR, F. 1962. Germination value: An Index Combining Speed and Completeness of Pine Seed Germination". Forest. Sci. 8(4):386-396.

16. DAVIDSON, R.; EDWARDS, D.; SZIKLAI, O.; EL-KASSABY, Y. 1996. Genetic variation in germination parameters among populations of pacific silver fir. Silvae Genetica. 45(2-3):165-171.

17. DIAS, J.; CAPRONI, A.; WADT, P.; SILVA, L.; TAVELLA, L.; OLIVEIRA, J. 2009. Quebra de dormência em diásporos de teca (Tectona grandis L.f.). Acta Amaz. 39(3):549-554.

18. EDWARDS, D. 1980. Maturity and quality of tree seeds a state-of-the-art review. Seed Sci.Techn. 8:625-657.

19. FERREIRA, C.D.; SOUTO, P.C.; LÚCIO, A.F.; SOUTO, J.S; SOUZA, B.V. 2012. Avaliações biométricas e germinação de sementes de Coaçu (Triplaris surinamensis Cham). Rev. Bras. Tecn. Apli. Ciên. Agrá. 5(1):147-162.

20. FIGLIOLIA, M.; OLIVEIRA, E.; PIÑA-RODRIGUES, F. 1993. Análise de sementes. En: Aguiar, I., Piña-Rodrigues, F., Figliolia, M. eds. Sementes florestais tropicais. Ed. Abrates, (Brasília). p.137-174.

21. FOFANA, I.; OFORI, D.; POITEL, M.; VERHAEGEN, D. 2009. Diversity and genetic structure of teak (Tectona grandis L.f) in its natural range using DNA microsatelite markers. New Forests. 37(1):175-195.

22. FONSECA, W. 2004. Manual Para Productores de Teca (Tectona grandis L.f) en Costa Rica. Heredia, Costa Rica. 121p.

23. GALLO, C.; ENRICO, J.M.; CRAVIOTTO, R.; ARANGO, M. 2012. Variabilidad de la viabilidad y vigor de lotes de semillas de soja con presencia de simientes verdes pertenecientes a cultivares de distintos grupos de maduración producidos en dos fechas de siembra. Rev. Invest. Agropec. 38(2):133-140.

24. HOPPE, J.; GENRO, C.; VARGAS, C.; FLORIANO, E.; REIS, E.; FORTES, F.; MÜLLER, I.; FARIAS, J.; CALEGARI, L.; DA COSTA, L. 2004. Produção de se- 
mentes e mudas florestais, Caderno Didático $\mathrm{n}^{\circ} 1$, $2^{a}$, UFSM-PPGEP, Santa Maria, 388p.

25. HUERTA-PANIAGUA, R.; RODRÍGUEZ-TREJO, D. 2011. Efecto del tamaño de semilla y la temperatura en la germinación de Quercus rugosa Née. Rev. Chapingo Serie Ciencias Forestales Ambiente. 17(2):179-187.

26. INDIRA, E.; BASHA, S.; CHACKO, K. 2000. Effect of seed size grading on the germination and growth of teak (Tectona grandis) seedlings. J. Trop. Forest Sci. 12(1):21-27.

27. INTERNATIONAL RULES FOR SEED TESTING -ISTA-. 2009. Edición 2005. International Seed Testing Association, Bassersdorf, Suiza. 363p.

28. KHURANA, E.; SINGH, J. 2001. Ecology of tree seed and seedlings: Implications for tropical forest conservation and restoration. Current Sci. 80(6):748-757.

29. LEISHMAN, M.; WRIGHT, I.; MOLES, A.; WESTOBY, M. 2000. The evolutionary ecology of seed size. En: Fenner, M. (ed). Seeds: The Ecology of Regeneration in Plant Communities. CABI Pub. London. p.31-57.

30. LO, E.; STEFANOVI, S.; RITLAND, K.; DICKINSON, T. 2010. Fine-scale comparisons of genetic variability in seed families of asexually and sexually reproducing Crataegus (Hawthorn; Rosaceae). Am. J. Bot. 97(6):1014-1024.

31. MAGUIRE, D. 1962. Speed of germination-aid. In: Selection and evaluation for seedling emergence and vigour. Crop Sci. 2:176-177.

32. MORENO, F.; PLAZA, G.; MAGNITSKIY, S. 2006. Efecto de la testa sobre la germinación de semillas de caucho (Hevea brasiliensis Muell.). Agr. Col. 24(2):290295.

33. MURILLO, O. 1998. Variación en parámetros de germinación de una población natural de Alnus acuminata de Guatemala e implicaciones para los lotes de semilla mezclada. Boletín de Semillas y Mejoramiento Genético Forestal. (CATIE, Costa Rica) 19:4-8.

34. MURILLO, O.; ESPITIA, M.; CASTILLO, C. 2012. Fuentes semilleras para la producción forestal. Universidad de Córdoba. Ed. Damar S.A.S (Bogotá). 176p.

35. NAMKOONG, G. 1997. A gene conservation plan for loblolly pine. Canadian J. Forest Res. 27(3):433-437.
36. NIEMBRO, A.; RAMÍREZ-GARCÍA, E.; APARICIO-RENTERÍA, A. 2007. Correlación entre características de frutos de Swietenia macrophylla King con su contenido de semillas desarrolladas. Foresta Veracruzana. 9(1):49-53.

37. PALENCIA, G.; MERCADO, T.; COMBATT, E. 2006. Estudio agroclimático del departamento de Córdoba. Universidad de Córdoba. Ed. Gráficas el Caribe (Montería).126p.

38. PASTORINO, M.; GALLO, L. 2000. Variación geográfica en peso de semilla en poblaciones naturales argentinas de "Ciprés de la Cordillera". Bosque 21(2):95-109.

39. REGO, F.; COSTA, R.; CONTINI, A.; MORENO, R.; RONDELLI, K.; KUMIMOTO, H. 2005. Variabilidade genética e estimativas de herdabilidade para o caráter germinação em matrizes de Albizia lebbeck. Ciência Rural. 35(5):1209-1212.

40. RESENDE, M.; ARAUJO, A.; SAMPAIO, P.; WIECHETECK, M. 1995. Acurácia seletiva, intervalos de confiança e variância de ganhos genéticos associados a 22 métodos de seleção em Pinus caribaea var. hondurensis. Rev. Floresta. 24(1):35-45.

41. RESENDE, M. 2006. O Software Selegen-Reml/Blup. Documentos EMBRAPA, Campo Grande. 305p.

42. RESENDE, M. 2007. Matemática e estatística na análise de experimentos e no melhoramento genético. EMBRAPA, Florestas, 362p.

43. RESENDE, M.; DUARTE, J. 2007. Precisão e controle de qualidade em experimentos de avaliação de cultivares. Pesq. Agrop. Trop. 37(3):182-194.

44. ROCHA, R.; VIEIRA, A.; SPINELLI, V.; VIEIRA, J. 2011. Caracterização de fatores que afetam a germinação de teca (Tectona grandis): temperatura e escarificação. Rev. Árvore, Viçosa-MG. 35(2):205-212.

45. RODRÍGUEZ-RIVAS, G.; MÁRQUEZ, J.; REBOLLEDOCAMACHO, 2001. Determinación del potencial y eficiencia de producción de semillas de Cedrela odorata L. y su relación con caracteres morfométricos de frutos. Foresta Veracruzana. 3(1):23-26.

46. SCHUHLI, G.; PALUDZYSZYN FILHO, E. 2010. O cenário da silvicultura de teca e perspectivas para o melhoramento genético. Pesq. Florestal Bras. Colombo. 30(63):217-230. 
47. SHIMELIS, H.; SHIRINGANI, R. 2010. Variance components and heritabilities of yield and agronomic traits among cowpea genotypes. Euphytica. 176(3):383389.

48. SILVA, K.B.; ALVES, E.U.; MATOS, V.P.; BRUNO, R.L. 2012. Caracterização morfológica de frutos, sementes e fases da germinação de Pachira aquatica Aubl. (Bombacaceae). Rev. Ciênc. Agr. 33(3):891-898.

49. STEEL, R.; TORRIE, J. 1980. Principles and procedures of statistics. McGraw-Hill. New York, United States. 633p.

50. SURI, S. 1984. Analytical study of teak provenance tests in North Raipur Division of Madhya Pradesh. Indian Forester. 110(4):345-363.

51. TENORIO-GALINDO, G.; RODRÍGUEZ-TREJO, D.; LÓPEZ-RÍOS, G. 2008. Efecto del tamaño y color de la semilla en la germinación de Cecropia obtusifolia Bertol (Cecropiaceae). Agrociencia. 42:585-593.
52. VALLEJO, F.; ESPITIA, M.; ESTRADA, E.; RAMÍREZ, H. 2010. Genética vegetal. Ed. U. N. Colombia, sede Palmira (Palmira). 384p.

53. VARGHESE, M.; NICODEMUS, A.; NAGARAJAN, B.; LINDGREN, D. 2006. Impact of fertility variation on gene diversity and drift in two clonal seed orchards of teak (Tectona grandis Linn. f.). New Forests. 31(3):497-512.

54. VENABLE, D.; PAKE, C. 1999. Population ecology of desert plans. In: Ecology od Desert Plants. Robichaux R.H. (ed). The University of Arizona Press. p.115142.

55. VIEIRA, A.; ROCHA, R.; REBELO, A. 2009. Avaliação de métodos para a superação de dormência de diásporos de teca (Tectona grandis L.f.). Floresta. Curitiba, PR. 39(2):273-278.

Recibido: Marzo 11 de 2013

Aceptado: Octubre 1 de 2013

Como citar:

Correa, E.; Espitia, M.; Araméndiz, H.; Murillo, O.; Pastrana, I. 2013. Variabilidad genética en semillas de árboles individuales de Tectona grandis L.f. En la conformación de lotes mezclados en córdoba, colombia. Rev. U.D.C.A Act. \& Div. Cient. 16(2): 379-389. 